九州大学学術情報リポジトリ

Kyushu University Institutional Repository

\title{
Wavelet analyses using parallel computing for plasma turbulence studies
}

Fuj isawa, Akihide

Research Institute for Applied Mechanics, Itoh Research Center for Plasma Turbulence, Kyushu University

Shimizu, Akihiro

National Institute for Fusion Science

Itoh, Kimitaka

National Institute for Fusion Science

Nagashima, Yoshihiko

Graduate School of Frontier Sciences, University of Tokyo

他

http://hdl. handle. net/2324/26366

出版情報: Physics of Plasmas. 17 (10)，pp.104503(1)-104503(3)，2010-10. American Institute of Physics

バージョン :

権利関係: (C) 2010 American Institute of Physics 
Wavelet analyses using parallel computing for plasma turbulence studies

A. Fujisawa, A. Shimizu, K. Itoh, Y. Nagashima, T. Yamada et al.

Citation: Phys. Plasmas 17, 104503 (2010); doi: 10.1063/1.3488244

View online: http://dx.doi.org/10.1063/1.3488244

View Table of Contents: http://pop.aip.org/resource/1/PHPAEN/v17/i10

Published by the American Institute of Physics.

\section{Related Articles}

Fast visible imaging and edge turbulence analysis in QUEST

Rev. Sci. Instrum. 83, 10E524 (2012)

Electromagnetic fluctuation spectrum associated with the drift Alfvén-cyclotron instability

Phys. Plasmas 19, 072318 (2012)

Revisited global drift fluid model for linear devices

Phys. Plasmas 19, 072317 (2012)

Electrostatic transport in L-mode scrape-off layer plasmas of Tore Supra tokamak. II. Transport by fluctuations Phys. Plasmas 19, 072314 (2012)

Effect of stochasticity in mean field dynamo models

Phys. Plasmas 19, 072310 (2012)

\section{Additional information on Phys. Plasmas}

Journal Homepage: http://pop.aip.org/

Journal Information: http://pop.aip.org/about/about_the_journal

Top downloads: http://pop.aip.org/features/most_downloaded

Information for Authors: http://pop.aip.org/authors

\section{ADVERTISEMENT}

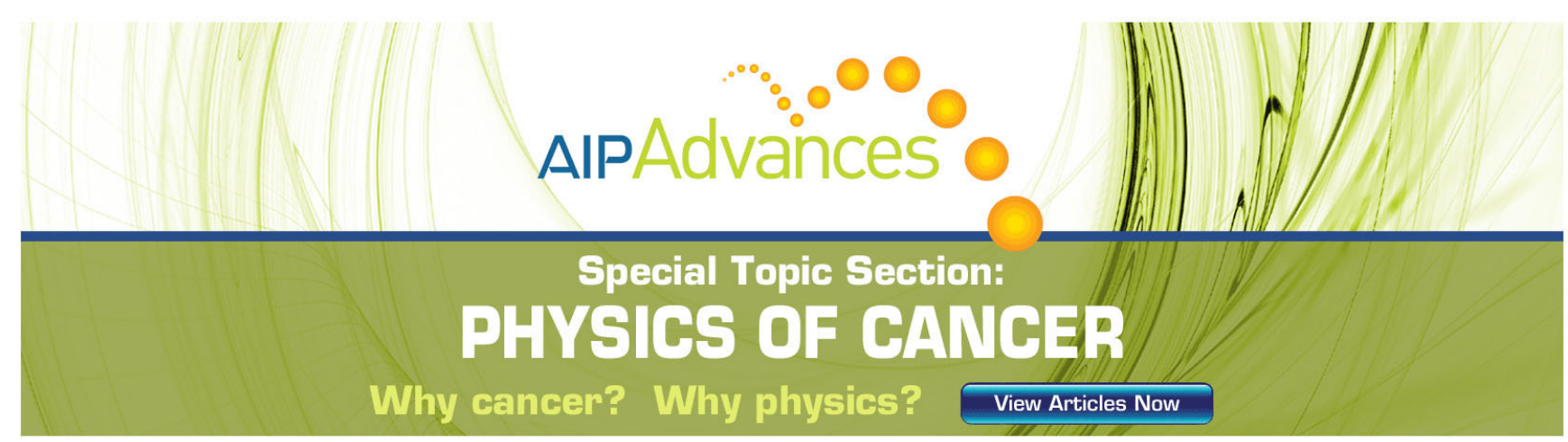




\title{
Wavelet analyses using parallel computing for plasma turbulence studies
}

\author{
A. Fujisawa, ${ }^{1}$ A. Shimizu, ${ }^{2}$ K. Itoh, ${ }^{2}$ Y. Nagashima, ${ }^{3}$ T. Yamada, ${ }^{3}$ S. Inagaki, ${ }^{1}$ K. Matsuoka,${ }^{1}$ \\ and S.-I. Itoh ${ }^{1}$ \\ ${ }^{1}$ Research Institute for Applied Mechanics and Itoh Research Center for Plasma Turbulence, \\ Kyushu University, Kasuga-kouen, Kasuga 816-8580, Japan \\ ${ }^{2}$ National Institute for Fusion Science, Oroshi-cho, Toki-shi 509-52, Japan \\ ${ }^{3}$ Graduate School of Frontier Sciences, The University of Tokyo, 5-1-5 Kashiwanoha, Kashiwa-shi, \\ Chiba 277-8561, Japan
}

(Received 19 July 2010; accepted 20 August 2010; published online 20 October 2010)

\begin{abstract}
The wavelet analyses have been carried out, using a cluster of personal computer, on the signal of electric field fluctuations measured with heavy ion beam probes in the compact helical system stellarator. The results have revealed the intermittent characteristics of turbulence and of the nonlinear couplings between elemental waves of turbulence. The usage of parallel computing is found to successfully reduce the calculation time as inversely proportional to the CPU number used the cluster, which shows the nature of "embarrassingly parallel computation." The present example of the wavelet analyses clearly demonstrates the importance of the advanced analyzing methods and the parallel computation for the modern studies of plasma turbulence. (C) 2010 American Institute of Physics. [doi:10.1063/1.3488244]
\end{abstract}

The understanding of turbulence should be a key issue for realizing magnetically confinement nuclear fusion, since the turbulence governs the plasma transport property to determine the plasma performance. ${ }^{1,2}$ Recently, the advanced methods to analyze turbulence, such as wavelet and bicoherence, have been developed to succeed in quantifying the nonlinear nature of turbulence, e.g., intermittency and mutual couplings between elemental waves or vortices. ${ }^{3,4}$ In fact, the wavelet and the wavelet bicoherence analyses succeeded in proving the couplings between zonal flows and background turbulence. ${ }^{5,6}$

For further understanding of turbulence, many other advanced analyses should be needed to reveal hidden natures of turbulence, although they should require a huge amount of processes or steps to often impose intolerable calculation time on a computer. Therefore, the modern experimental studies of turbulence need the most excellent capability of computers. The purpose of this article is to demonstrate the necessity of advanced analysis of plasma turbulence and the excellence of the parallel computing for experimental turbulence studies, by presenting a set of wavelet analyses applied on the electric field fluctuations in the compact helical system (CHS).

CHS is a toroidal device of medium size, $R=1.0 \mathrm{~m}$ and $\bar{a}=0.2 \mathrm{~m}$, equipped with two heavy ion beam probes (HIBP), where $R$ and $\bar{a}$ are the major and averaged minor radius, respectively. The analyzed electric field fluctuations are measured with the HIBPs on a CHS plasma produced with electron cyclotron resonance heating (ECRH) and the confinement magnetic field strength of $0.9 \mathrm{~T}$. The HIBP data are sampled with digitizers named WE7116, a module of Yokogawa WE series. A channel of the digitizer has 2M words memories, and the maximum sampling rate can be up to $25 \mathrm{MHz}$. The sampling rate in the experiment was set at 5 (or 10) $\mathrm{MHz}$ to cover the whole duration of CHS discharges. Correspondingly, the Nyquist frequency here is 2.5 (or 5)
$\mathrm{MHz}$ in this series of experiments. Electric field measurement needs 16 channels at a plasma spatial point, hence, a total of 32 Mbyte words data are managed for the analyses presented here. The cluster of personal computers used for the analysis is constructed with a dozen of Macintosh Xserve Quad Intel Xeon, each of which is equipped with four CPUs with the clocks of $2.26 \mathrm{GHz}$, hence, the system owns 40 CPUs in total.

The wavelet transformation adopted here is defined as

$$
\widetilde{g}(f, t)=\int_{-\infty}^{\infty} G(\tau) \Phi(f, t-\tau) d \tau,
$$

with $\Phi(f, t)=\sqrt{f} \exp \left[i 2 \pi f t-(f t)^{2} / 2\right]$, which has a natural correspondence with the traditional Fourier transformation (FFT). In addition, the square $|\widetilde{g}(f, t)|^{2}$ means the spectral power density at a frequency and time, $(f, t)$. This property of the wavelet transformation allows us to evaluate the intermittent nonlinear couplings between elemental waves. As is similar to the bicoherence based on the Fourier transformation, ${ }^{7,8}$ the squared wavelet bicoherence ${ }^{6,9}$ is defined as a simple extension of the traditional form as

$$
b^{2}\left(\omega_{1}, \omega_{2}\right)=\frac{\left|\int \widetilde{g}_{i}\left(\omega_{1}, t\right) \widetilde{g}_{i}\left(\omega_{2}, t\right) \widetilde{g}_{i}^{*}\left(\omega_{3}, t\right) d t\right|^{2}}{\int\left|\widetilde{g}_{i}\left(\omega_{1}, t\right) \widetilde{g}_{i}\left(\omega_{2}, t\right)\right|^{2} d t \int\left|\widetilde{g}_{i}\left(\omega_{3}, t\right)\right|^{2} d t} .
$$

In the definition of the bicoherence, the frequencies of three waves should satisfy the frequency matching condition $f_{1}+f_{2}=f_{3}$.

Figure 1 shows the results of the wavelet analyses applied on the HIBP data. The temporal evolution of the mean wavelet power density is shown in Fig. 1(a). The temporal evolution is calculated up to $500 \mathrm{kHz}$ every $1 \mathrm{kHz}$ for 120 ms every $50 \mu \mathrm{s}$, therefore, the total number of the spectral points calculated in $(t, f)$-domain is $2400 \times 500=1.2 \times 10^{6}$, according to the following equation as: 


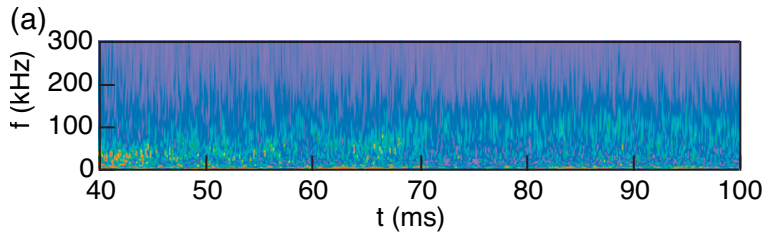

(b)

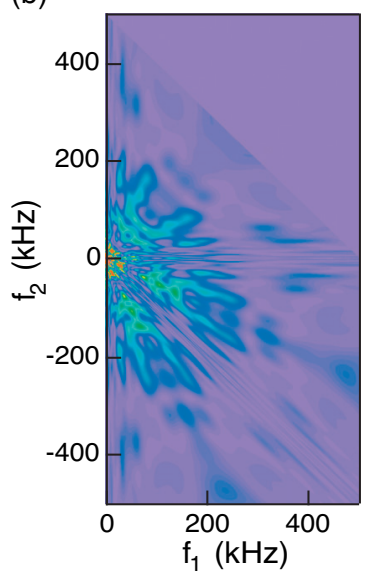

(c)

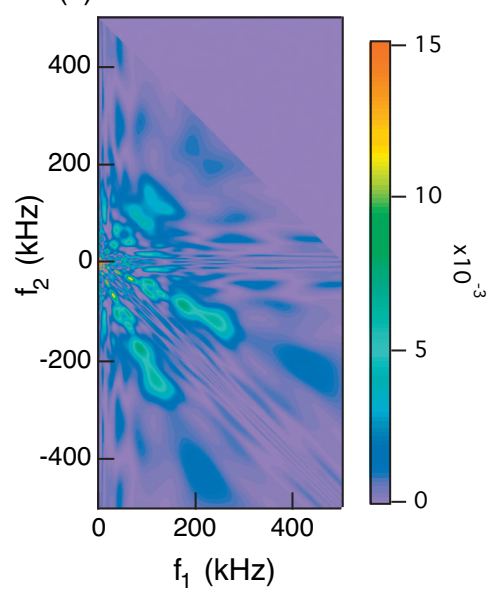

FIG. 1. (Color) An example of wavelet analysis. (a) Temporal evolution of wavelet spectral power, (b) bicoherence diagrams from 42 to $62 \mathrm{~ms}$, and (c) that of 70-90 ms.

$$
\widetilde{W}(f, t)=\frac{1}{2 T} \int_{t-T}^{t+T}|g(f, \tau)|^{2} d \tau,
$$

setting $T=50 \mu \mathrm{s}$. In the case of Fig. 1(a), the sampling rate (time) is chosen as $5 \mathrm{MHz}$ (200 ns) and each averaged spectral power is evaluated from 500 temporal points around it. The total time necessary for the calculation is $2340 \mathrm{~s}$ using 40 CPUs (or each CPU takes charge in calculating $3 \mathrm{~ms}$ data). The calculation time is measured using the function "gettimeofday_sec" in C.

In the evolution of the wavelet power density [Fig. 1(a)], it is likely that the turbulence characteristics may change around $t \simeq 65 \mathrm{~ms}$. The bicoherence diagrams shown in Figs. 1(b) and 1(c) are calculated, according to Eq. (2), for these two characteristic periods of the discharge, $t=42-62 \mathrm{~ms}$ and $t=70-90 \mathrm{~ms}$, respectively. In comparison the two bicoherence diagrams appear different, suggesting clear changes in the manners of nonlinear couplings in temporal average; in the early phase higher bicoherence can be seen in the regions along the lines of $f_{2} \sim 3 f_{1}, f_{2} \sim 3 f_{1} / 2, f_{2} \sim 2 f_{1}, f_{2} \sim 2 f_{1} / 3$, $f_{2} \sim f_{1} / 2$, and $f_{2} \sim f_{1} / 3$, while in the later phase the high bicoherence remains along those of $f_{2} \sim 2 f_{1}$ and $f_{2} \sim f_{1} / 2$.

In the usual bicoherence analysis based on FFT, the analyzing period of $20 \mathrm{~ms}$ is completely insufficient to resolve the difference between the above two phases. ${ }^{10}$ However, the wavelet bicoherence can succeed in visualizing the difference in the turbulence characteristics to show its superiority as a turbulence analyzing tool. Here, the wavelet spectral density was evaluated for the temporal window of $20 \mathrm{~ms}$ every $200 \mathrm{~ns}$ and for the frequency up to $500 \mathrm{kHz}$ every $1 \mathrm{kHz}$, therefore, the number of the temporal realizations is $1 \times 10^{5}$. Therefore, the total number of the necessary integration is $5 \times 10^{7}$. Each CPU is assigned to completing the cal-

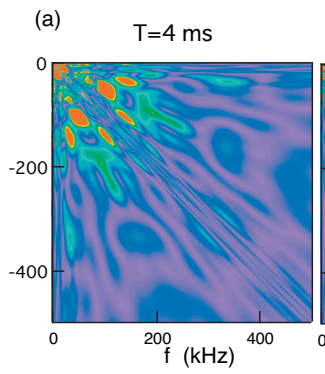

(b)

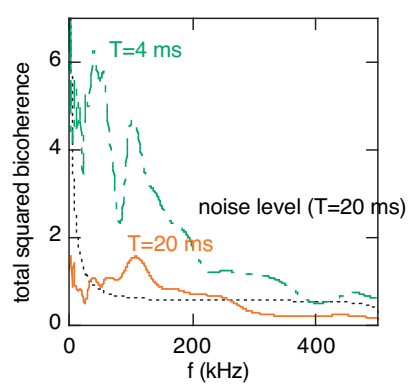

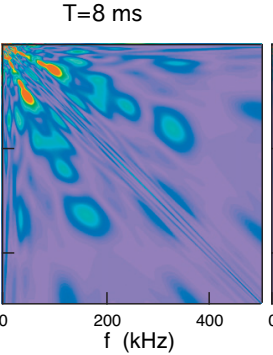

(c)

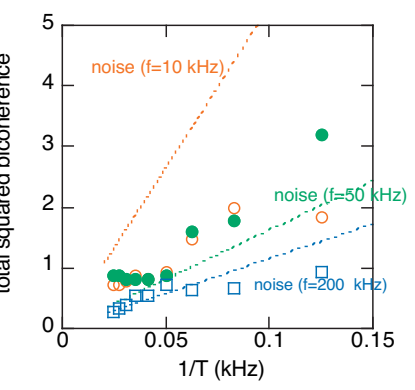

FIG. 2. (Color) (a) Comparison between bicoherence diagrams of three periods, (left) 70-74 ms, (middle) 70-78 ms, and (right) 70-94 ms. (b) Total bicoherence calculated for the period of $20 \mathrm{~ms}$ as a function of frequency, with the one of the period of $4 \mathrm{~ms}$ for reference. The dashed line indicates the noise level evaluated using Milligen's formula. (c) The convergence property of the total bicoherence at several frequencies, 10 (circles), 50 (closed circles), and $200 \mathrm{kHz}$ (squares). The evaluated total bicoherence is changed as a function of the inverse of the calculation period. The dashed lines represent the change of the noise level.

culation for a temporal domain. In the case of 40 CPUs used (or that the assigned temporal period of the calculation is $0.5 \mathrm{~ms}$ ), the necessary calculation time is $278 \mathrm{~s} \sim 4.6 \mathrm{~min}$.

The discussion should be made on the significance and convergence of the bicoherence values obtained in the wavelet analysis. Figure 2(a) shows the bicoherence diagrams for the three different period of 70-74, 70-78, and 70-94 ms. The comparison between these three diagrams, assuming that the turbulence characteristics are not changed for 70-94 ms,

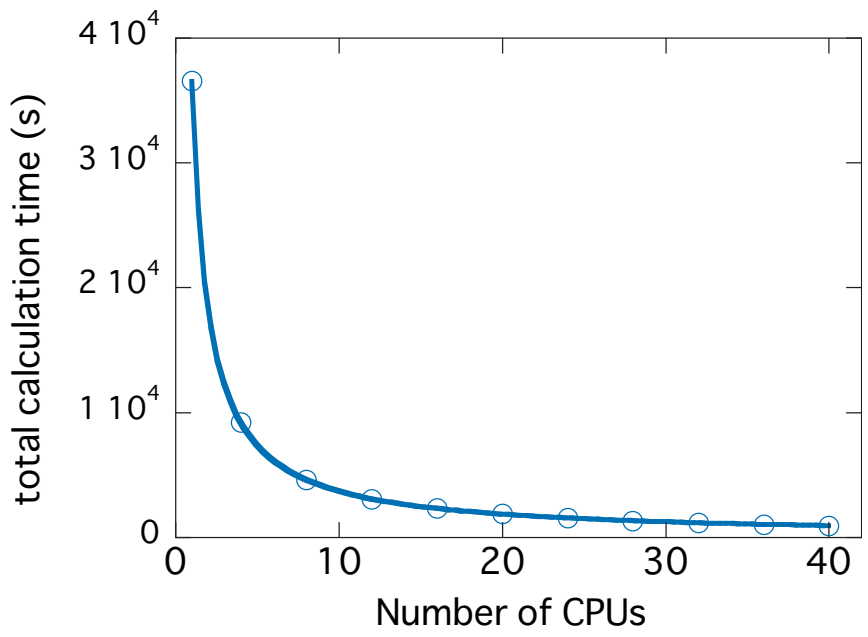

FIG. 3. (Color) The necessary computation time as a function of the number of CPUs in a cluster. In case of a CPU used, the necessary time is $36551 \mathrm{~s} \sim 10 \mathrm{~h}$. The necessary time is reduced by almost $1 / N$, where $N$ is the number of CPUs used in parallel computing. 
can address the convergence property of the bicoherence. After a longer temporal average, intermittent properties in the couplings should drop and the temporally averaged couplings should remain; i.e., the couplings should be constantly strong on the lines of $f_{1} \sim 2 f_{2}$ and $f_{2} \sim 2 f_{1}$ in the later phase of the discharge.

Figure 2(b) shows the comparison between the total bicoherence and the noise level as a function of frequency, for the case of bicoherence analysis of the period of 70-90 ms [or Fig. 1(c)], where the noise level is evaluated according to Milligen's formula. ${ }^{9}$ For reference, the dashed-dotted line shows the total bicoherence calculated for the period of 70-74 ms. Compared to the noise level, the significant stationary couplings are found in the frequency range of $\sim 50-150 \mathrm{kHz}$, for the $20 \mathrm{~ms}$ average, while the number of realizations (or the temporal periods) are insufficient to find the stationary bicoherence in the lower $(f<50 \mathrm{kHz})$ and higher $(f>200 \mathrm{kHz})$ frequency ranges.

Figure 2(c) shows the convergence properties of the bicoherence values at several frequencies, 10, 50, and $200 \mathrm{kHz}$, as a function of the inverse of the calculation period, $1 / T$. As is similar to the above conclusion, the significant coupling is found in the case of $f=50 \mathrm{kHz}$, while the cases of $f=10$ and $200 \mathrm{kHz}$ need more effective realizations (or more calculation time) to find a significant coupling. The lack of significant bicoherence at low frequency around $f \simeq 10 \mathrm{kHz}$ should be ascribed to insufficiency of the analyzing periods to resolve such low frequency, while that at high frequency above $f \simeq 200 \mathrm{kHz}$ should be to the weak coupling in this high frequency region.

Finally, it is worth a while to discuss the efficiency of the parallel computing. In order to confirm the efficiency of parallel computing, the calculation time to obtain the bicoherence diagram for $20 \mathrm{~ms}$ is shown in Fig. 3 as a function of the number of CPUs in the cluster. In this case, the sampling rate (time) is chosen as $10 \mathrm{MHz}(100 \mathrm{~ns})$. The calculation time using 40 CPUs is $934 \mathrm{~s}$, which is about four times longer than the previous cases in Figs. 1(b) and 1(c). The result obviously demonstrates that the calculation time is inversely proportional to the number of the CPUs. In addition, the trials show that the time necessary for transferring the data between the cluster computers, roughly less than $0.5 \mathrm{~s}$, is negligible for total calculation time. In other words, the time for data transfer becomes significant if the number of CPUs becomes comparable to approximately 70000 .

In conclusion, the wavelet analyses performed on the electric field fluctuations in CHS, using the parallel computing, revealed the clear change of the manner of three wave couplings, which cannot be resolved by traditional Fourier spectral analysis. The usage of the personal computer cluster makes the calculation time tolerable and allowing frequent tries and errors in the turbulence analyses of ultimate conditions. For example, the significant wavelet bicoherence may be obtained even in the low or high frequency $(f \simeq 10 \mathrm{kHz}$ and $f>200 \mathrm{kHz}$ in this article) where the signal cannot be distinguished from the noise level in a reasonable calculation time, if a sufficient longer period of stationary turbulence is available. Besides, for future investigation of plasma turbulence, the bispectral analysis widely carried out at present should proceed to higher-order analysis, like trispectrum, ${ }^{11}$ to clarify the multiple wave couplings, which should need tremendous steps of calculations. Moreover, multipoints measurements are essential to cover a wide range of space for resolving the wavenumbers in addition to frequency, ${ }^{12-14}$ since the turbulence is a phenomenon varying widely in space and time. The development of the methods using parallel computing becomes absolutely indispensable for the preparation of such future studies of turbulence.

The authors would like to expresses their sincere appreciation to Professor H. Zushi and the members of AFRC for their cooperation. This work is partially supported by Grantin-Aids for Scientific Research (Grant Nos. 21224014 and 18360447).

${ }^{1}$ P. C. Liewer, Nucl. Fusion 25, 543 (1985).

${ }^{2}$ A. J. Wootton, B. A. Carreras, H. Matsumoto, K. McGuire, W. A. Peebles, Ch. P. Ritz, P. W. Terry, and S. J. Zweben, Phys. Fluids B 2, 2879 (1990).

${ }^{3}$ A. Fujisawa, Nucl. Fusion 49, 013001 (2009).

${ }^{4}$ G. R. Tynan, A. Fujisawa, and G. McKee, Plasma Phys. Controlled Fusion 51, 113001 (2009).

${ }^{5}$ A. Fujisawa, A. Shimizu, H. Nakano, S. Ohshima, K. Itoh, Y. Nagashima, S-I. Itoh, H. Iguchi, Y. Yoshimura, T. Minami, K. Nagaoka, C. Takahashi, M. Kojima, S. Nishimura, M. Isobe, C. Suzuki, T. Akiyama, T. Ido, K. Matsuoka, S. Okamura, and P. H. Diamond, J. Phys. Soc. Jpn. 76, 033501 (2007).

${ }^{6}$ A. Fujisawa, A. Shimizu, H. Nakano, S. Ohshima, K. Itoh, Y. Nagashima, S.-I. Itoh, H. Iguchi, Y. Yoshimura, T. Minami, K. Nagaoka, C. Takahashi, M. Kojima, S. Nishimura, M. Isobe, C. Suzuki, T. Akiyama, T. Ido, K. Matsuoka, S. Okamura, and P. H. Diamond, Plasma Phys. Controlled Fusion 49, 211 (2007).

${ }^{7}$ Y. C. Kim and E. J. Powers, IEEE Trans. Plasma Sci. 7, 120 (1979).

${ }^{8}$ Y. Nagashima, K. Hoshino, A. Ejiri, K. Shinohara, Y. Takase, K. Tsuzuki, K. Uehara, H. Kawashima, H. Ogawa, T. Ido, Y. Kusama, and Y. Miura, Phys. Rev. Lett. 95, 095002 (2005).

${ }^{9}$ B. Ph. van Milligen, C. Hidalgo, and E. Sánchez, Phys. Rev. Lett. 74, 395 (1995).

${ }^{10}$ Y. Nagashima, S.-I. Itoh, M. Yagi, K. Itoh, and A. Fujisawa, Rev. Sci. Instrum. 77, 045110 (2006).

${ }^{11}$ W. B. Collis, P. R. White, and J. K. Hammond, Mech. Syst. Signal Process. 12, 375 (1998).

${ }^{12}$ A. Latten, T. Klinger, A. Piel, and Th. Pierre, Rev. Sci. Instrum. 66, 3254 (1995).

${ }^{13}$ F. Brochard, T. Windisch, O. Grulke, and T. Klinger, Phys. Plasmas 13, 122305 (2006).

${ }^{14}$ T. Yamada, S-I. Itoh, T. Maruta, N. Kasuya, Y. Nagashima, S. Shinohara, K. Terasaka, M. Yagi, S. Inagaki, Y. Kawai, A. Fujisawa, and K. Itoh, Nat. Phys. 4, 721 (2008). 